\title{
Temperature oscillations of hydrothermal waves in thermocapillary-buoyancy convection
}

\author{
Miguel Angel Pelacho and Javier Burguete \\ Departamento de Física y Matemática Aplicada, Facultad de Ciencias, Universidad de Navarra, 31080 Pamplona, Navarra, Spain
}

(Received 4 May 1998)

\begin{abstract}
We report an experimental study of a fluid layer of Prandtl number $\operatorname{Pr}=10.3$ subject to a horizontal temperature gradient with the surface open to the air. For certain values of the control parameters (fluid depth and temperature difference) the basic flow is destabilized and a pair of so-called oblique hydrothermal waves is observed. It is found that some main physical features are in agreement with those analyzed by Smith and Davis [J. Fluid Mech. 132, 119 (1983)]. Vertical and horizontal temperature profiles were measured, showing bulk temperature oscillations that propagate in the direction of the temperature gradient. This behavior is similar to the instability mechanism explained by Smith and Davis for intermediate Pr.
\end{abstract}

[S1063-651X(99)08401-9]

PACS number(s): 47.20.Dr, 47.35.+i

\section{INTRODUCTION}

In recent years, interest in instabilities and pattern formation in convective systems has increased. Several studies have been devoted to the one-dimensional dynamics in thermocapillary-buoyancy convection. One of the convective systems of growing attention is lateral heating, which happens when a horizontal temperature gradient is imposed on a fluid layer and provides a situation where the steady convective basic flow can be destabilized. These systems also have technical interest, i.e., in solidification processes in silicon crystal growth, where the instabilities produced by temperature oscillations cause a defective crystal [1].

Smith and Davis [2] (SD) analyzed the linear stability of an infinite fluid layer of depth $h$ submitted to a constant horizontal temperature gradient. In this system, as soon as a temperature gradient is imposed, buoyancy and thermocapillary forces produce a convective circulation. In their analysis, buoyancy forces were not considered and only thermocapillarity was taken into account. Two different basic states can be distinguished: a return flow (a closed flow where the fluid on the surface goes from the hot to the cold sidewall and returns from the bottom) and a linear flow (an open flow where the fluid moves in a unique direction). In the former, two hydrothermal waves appear superimposed on the basic flow for a particular temperature gradient, propagating obliquely upstream, i.e., opposite to the surface flow.

Although thermogravitational effects were analyzed by Laure and Roux [3] on fluids of low Prandtl numbers Pr, subsequently some authors studied the linear stability when both buoyancy and thermocapillary effects are considered. In addition, Parmentier et al. [4] extended the range of Pr to 10 and found that their results agreed with SD's results if buoyancy forces were not taken into account, but they were different when these forces were considered.

Oscillatory modes, steady [5] and traveling, have been observed in various lateral heating experiments. Braunsfurth and Homsy [6], for $\mathrm{Pr}=4$, observed a transition from a twodimensional flow to a steady three-dimensional flow in a small square configuration (the ratio between both horizontal dimensions and the height of fluid was varied from 1 to 8 ). In the experiment of Daviaud and Vince [7] a transition from traveling waves to a stationary pattern was detected for a particular height $\left(h_{c}\right)$ for $\operatorname{Pr}=10$. At lower heights the system exhibits waves propagating almost perpendicular to the gradient direction and for greater heights stationary modes appeared. Schwabe et al. [8], using a fluid with $\operatorname{Pr}=17$, reported a first transition from one stationary roll to several stationary rolls and a second transition to two kinds of traveling waves observed in both rectangular and annular cavities. One of the instabilities had similar features to the traveling waves mentioned in Ref. [2]. For a larger Prandtl number $(\mathrm{Pr}=30)$ Ezersky et al. [9] found a wave number close to SD's value, but the propagating direction was opposite to that predicted. In a recent experiment performed by Riley and Neitzel [10] with $\operatorname{Pr}=13.9$, only a traveling wave was observed, such as is expected from the nonlinear analysis of Smith [11].

A theoretical and numerical work carried out by Mercier and Normand [12] using the same fluid properties as in Ref. [7] reveals a quantitative agreement for the physical features of the hydrothermal waves of Ref. [7]. They argue that lateral confinement in experiments could delay the onset of oscillatory instability. A more recent numerical study [13] shows a dependence of the oscillatory modes and their characteristics on the lateral confinement.

Several container configurations have been used in the experiments. In some of them, the length $l_{x}$ in the gradient direction was smaller than the distance between the other sides $\left(l_{y}\right)$ [7]. On the contrary, other cavities have $l_{x} \gg l_{y}$ [14]. The distances $l_{x}$ and $l_{y}$ of our container are much bigger than the depth of fluid $d\left(l_{x} \sim l_{y} \gg d\right)$ and we expect to avoid confinement effects due to the proximity of the walls.

Depending on whether the Prandtl number is small ( $\mathrm{Pr}$ $\approx 0$ ) or large $(\operatorname{Pr} \approx \infty), \mathrm{SD}[2]$ and Smith [15] distinguish two instability mechanisms that give rise to hydrothermal waves with different dynamical properties. In the case of small Pr, an initial surface hot spot induces an upward flow with a lower basic-state velocity toward the surface. As a result, an inertial force is created in the upstream direction driving a velocity perturbation. The colder upstream flow cools the hot spot and after a time it is eliminated. However, at that moment the upstream flow is still present so that the surrounding mean temperature is overshot. Now the new 
cold spot originates a flow toward the spot and a downflow beneath it. Nevertheless, again owing to the inertia, this cold spot is eliminated and a new hot spot is formed. In this mechanism the inertia effects produce the proper phasing between the streamwise flow and the vertical flow. The energy is extracted from the horizontal basic-state temperature field. As the flow in the streamwise direction is constrained by continuity, the proper phasing between it and the vertical flow cannot be attained and only the spanwise flow survives. Then a spanwise traveling hydrothermal wave appears.

When Pr is large, inertial effects are absent and so there is not a proper phasing between the streamwise flow and the vertical flow. Now the mechanism consists of convective heating in the interior of the fluid. A hot spot in the form of a line perpendicular to the gradient direction causes an interior hot spot to develop at mid-depth toward the hot side. The temperature of this interior hot spot becomes very large when the Marangoni number is large enough. Then the interior spot heats the surface, shifting upstream the original hot spot and producing hydrothermal waves propagating in the upstream direction.

According to SD, for an intermediate Pr a combination of these two mechanisms is present and the instability propagates upstream at some intermediate angle with respect to the gradient direction. In our experiment a fluid with Pr roughly 10, which could be considered intermediate according to other studies [9,7], is used and so both mechanisms can be expected. For Pr much larger than 10 other mechanisms based on the destabilization of the boundary layer seem to be the origin of another kind of instability [16].

Related to this, first we wondered whether instabilities such as oblique traveling waves appeared. Second, we wanted to determine experimentally the temperature field of the waves in order to obtain evidence of the instability mechanism of SD in a convective experiment.

In the next section we specify the details of the experimental setup. Results are given in Sec. III, and in Sec. IV we discuss and compare them with other results. Finally, we state some conclusions in Sec. V.

\section{EXPERIMENTAL SETUP}

The fluid, a silicon oil with $\operatorname{Pr}=10.3$ at $25^{\circ} \mathrm{C}$ and kinematic viscosity $\nu=0.65 \mathrm{cS}$, is placed between two lateral copper blocks and put into a closed container made of Plexiglas to reduce evaporation (see Fig. 1). For $h=2 \mathrm{~mm}$ the evaporated fluid was less than $1 \%$ per hour $(20 \mu \mathrm{m} / \mathrm{h})$ and thus we could not obtain temporal series longer than a few hours. We consider the $x$ axis as the direction opposite to the temperature gradient, the $y$ axis perpendicular to $x$, and the vertical direction marked by $z$.

Copper blocks are maintained at different temperatures $T_{+}$and $T_{-}$in such a way that the mean temperature inside the cell is the room temperature $T_{\text {room }}=22^{\circ} \mathrm{C}$. The maximum temperature difference $\left(\Delta T_{\max } \simeq 12 \mathrm{~K}\right)$ is small enough to ensure that the physical properties of the fluid are nearly constant (Boussinesq approximation). A water circulation at constant temperature goes through each copper block providing a stable temperature $( \pm 0.05 \mathrm{~K})$. Conductivity of the bottom plate $\left(\lambda=0.17 \mathrm{~W} \mathrm{~m}^{-1} \mathrm{~K}^{-1}\right)$ is similar to the fluid conductivity $\left(\lambda=0.1 \mathrm{~W} \mathrm{~m}^{-1} \mathrm{~K}^{-1}\right)$. The mass of
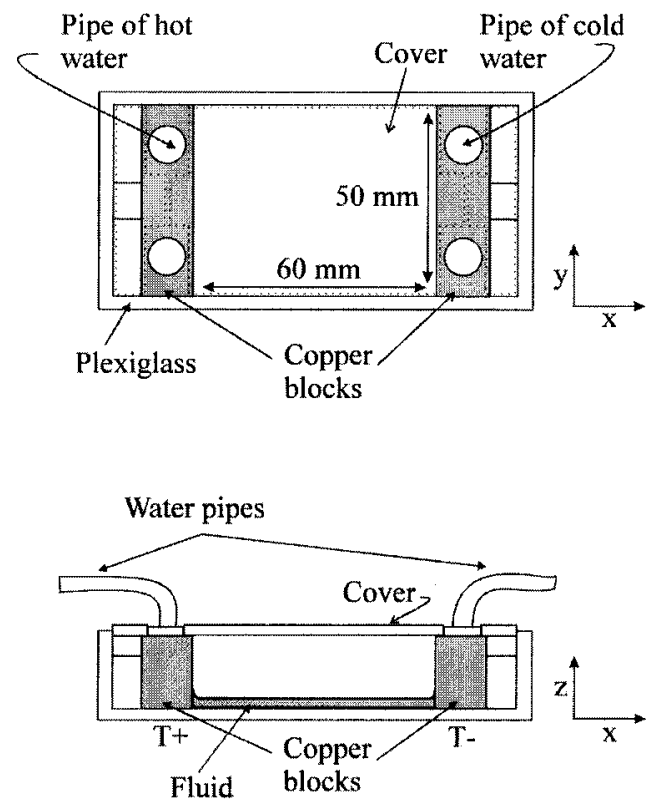

FIG. 1. Top view and front view of the experimental cell.

the copper pieces is big enough so that the temperature of the block is not disturbed by the temperature oscillations on the fluid. The distances between the blocks $l_{x}=60 \mathrm{~mm}$ and the other horizontal dimension $l_{y}=50 \mathrm{~mm}$ were kept constant. The range of the fluid depth was $1.25 \mathrm{~mm}<h<3.5 \mathrm{~mm}$, thus the aspect ratio $\left(\Gamma=l_{x} / h\right)$ had a value between 17 and 48.

Shadowgraph technique was used to capture images from the top of the cell using a charge-coupled device camera. The visualization field is limited to a central zone of 40 $\times 30 \mathrm{~mm}^{2}$ because of the meniscus. A line of pixels in both the $x$ and $y$ directions was periodically recorded in a computer to construct spatiotemporal diagrams. Using these images, the thresholds of the instabilities and some dynamical characteristics could be measured: wave number, frequency, and propagating angle of the waves. Nevertheless, these thresholds are overshot because the wave amplitude must be large enough to be detected over the noise and thus the patterns of small amplitude cannot be observed.

We carried out several temperature measurements that were started after a thermalization time of $2 \mathrm{~h}$. The horizontal temperature difference was measured with thermocouples placed in each copper block. For changes of the temperature of the extremes the thermalization time was of several minutes. This time is long compared to the characteristic thermal diffusion time $h^{2} / \kappa \simeq 64 \mathrm{~s}$.

As the aim of this work is to obtain the behavior of the temperature field into the fluid layer when a horizontal gradient is applied, several procedures were performed to measure this temperature. Its evolution in time has been registered by introducing a thermocouple into the fluid whose spatial position was controlled using a micrometer with an accuracy of $1 \mu \mathrm{m}$. Four thermocouples, aligned along $x$ and spaced $2.5 \mathrm{~mm}$ apart, were needed to obtain the temperature in various spatial positions on the gradient direction at the same time. Changing their vertical position, the fluid temperature was registered at several levels. The data acquisition time between one measurement given by a thermocouple and 


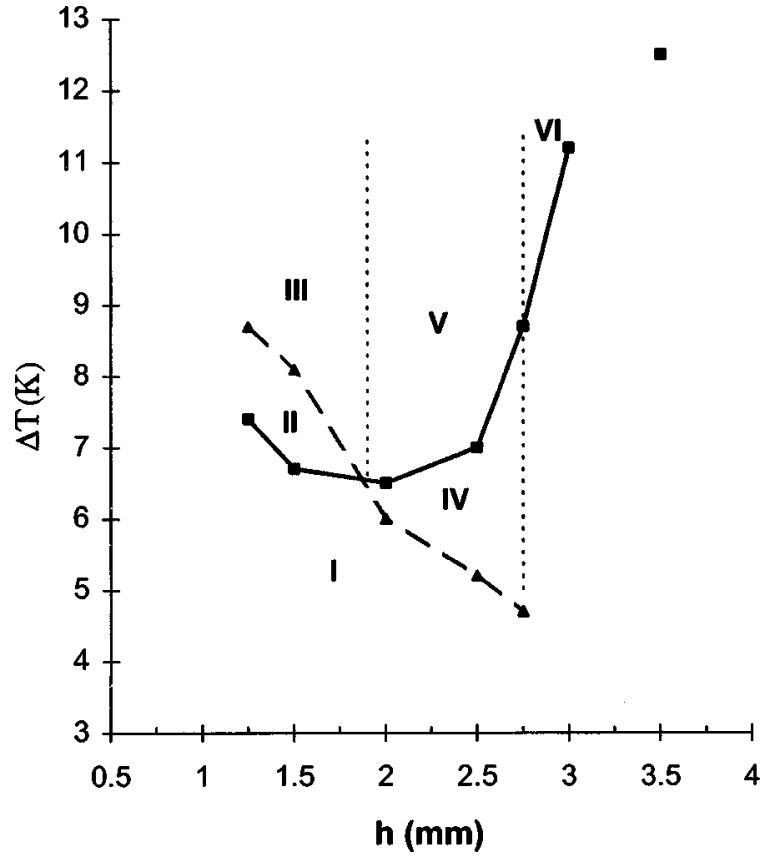

FIG. 2. Stability regions of oscillatory and stationary modes. The transition to traveling waves is represented by $\mathbf{\square}$ and the transition to rolls by $\boldsymbol{\Delta}$. Vertical discontinuous line divides regions III and $\mathrm{V}$ only for clarity.

the next was $0.1 \mathrm{~s}$, small enough considering the period of the oscillations observed.

\section{EXPERIMENTAL RESULTS}

According to the value of the parameters, the temperature difference $\Delta T$ between the copper blocks and fluid depth $h$, several regions of stability can be distinguished. The transitions between the stability regions were obtained from shadowgraphy (see Fig. 2).

(i) From $h=1.25 \mathrm{~mm}$ to $h<2 \mathrm{~mm}$. When $\Delta T$ is large enough, a transition from the basic flow (region I) to traveling waves (region II) takes place. For an increase of $\Delta T$ stationary rolls with their axes perpendicular to the gradient vector are seen superimposed on traveling waves (region III). Thus, in this region both instabilities are coexisting.

(ii) From $h=2 \mathrm{~mm}$ to $h=2.75 \mathrm{~mm}$. Basic flow is destabilized giving rise to stationary rolls perpendicular to the temperature gradient (region IV). The number of rolls decreases and their width increases as $h$ increases. For a higher $\Delta T$ a second transition to traveling waves is observed without the presence of rolls (region V).

(iii) From $h>2.75 \mathrm{~mm}$ to $h<3.5 \mathrm{~mm}$. Only waves were obtained (region VI).

Traveling instabilities in regions II, III, V, and VI present a similar behavior: a pair of waves traveling from the cold to the hot sidewall whose wave vector forms an angle with the temperature gradient (Fig. 3). For $h=2 \mathrm{~mm}$, the minimal $\Delta T$ at the threshold of the rolls was $6 \mathrm{~K}$ and the transition to traveling waves took place at $6.5 \mathrm{~K}$.

The transition from the basic flow to the stationary rolls is not well defined. The discontinuous line signals the minimum $\Delta T$ from which we can observe the rolls, in spite of the existence under this line of rolls with lower amplitude. For

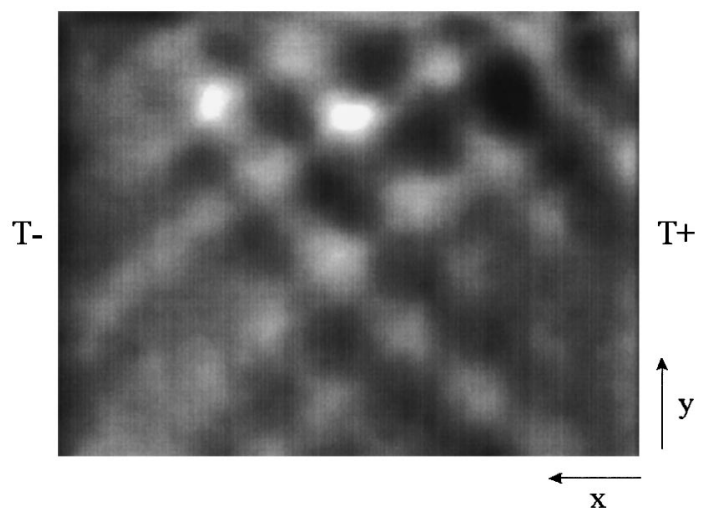

FIG. 3. Top image of the hydrothermal waves obtained by shadowgraph technique. Cold sidewall is indicated by $T-$ and hot sidewall by $T+$. Both of the two oblique waves are traveling from the cold to the hot side at the same time.

$h>2.75 \mathrm{~mm}$, only one roll fills the whole cell. For $h$ $=3.5 \mathrm{~mm}$ and higher, other traveling oscillations were seen at the same time as the first we previously observed, although the propagation direction was downstream and the wave vector and frequency were very different. These waves are more similar to those found in fluid with larger $\operatorname{Pr}$ [9]. For $h<1.25 \mathrm{~mm}$ we could not obtain a good image with our system due to the layer being too shallow.

The main physical properties, the frequency, wave number, and angle between the wave vector and gradient, were obtained at threshold for the parameter values through spatiotemporal diagrams. The magnitudes used to nondimensionalize were $h$ for length and $h^{2} / \nu$ for time. Thus the nondimensional wave number was almost constant at the average $K=2.2$ and the frequency varied according to depth between $4.1<\omega<15.9$. Both properties are shown in Figs. 4 and 5 .

The influence of the dimensions of the cell in the different dynamical behaviors observed in many experiments is still unknown. However, from some experiments [7,17] it seems that if the distance between heaters is shorter, waves are seen for a lower temperature difference. When this distance was $10 \mathrm{~mm}$, the minimal threshold was found to be between 3 and $4 \mathrm{~K}$ [7]. An increase to $30 \mathrm{~mm}$ gave rise to a higher temperature difference: $4 \mathrm{~K}$ [17]. We obtained another value

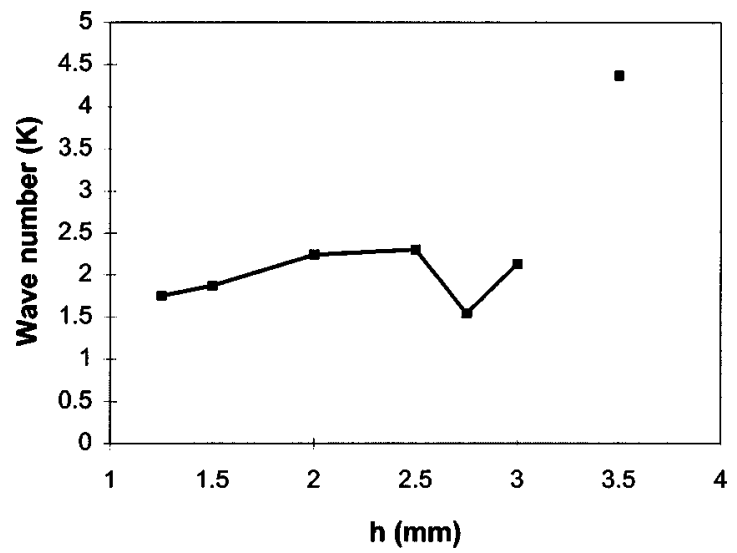

FIG. 4. Nondimensional wave number vs the fluid depth at the threshold of the waves. 


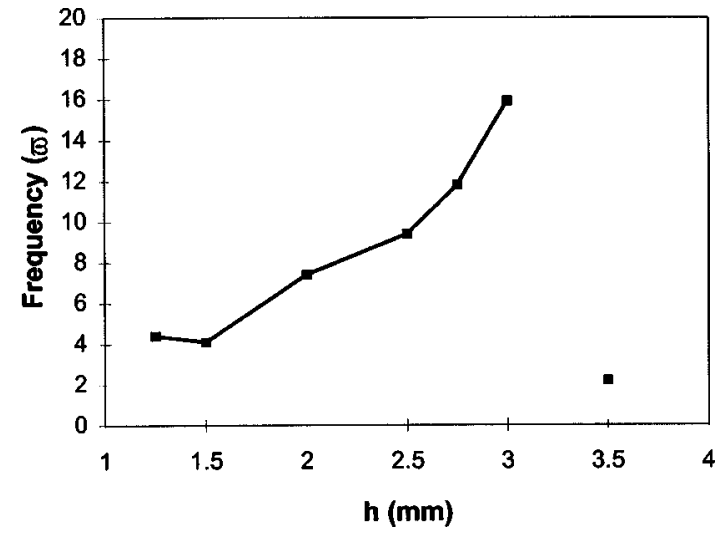

FIG. 5. Nondimensional frequency vs the fluid depth at the threshold of the waves.

of the temperature $(5 \mathrm{~K})$ when the length of our cell was changed to $40 \mathrm{~mm}$. Finally, for $l_{x}=60 \mathrm{~mm}$ the minimum temperature difference reached $6.5 \mathrm{~K}$ in our experiment. According to this result, a longer $l_{x}$ could mean that a higher $\Delta T$ is needed for the waves to be present and so both parameters govern the appearance of the waves.

Because of the relevance of the horizontal gradient in the experiment we defined two nondimensional parameters: the Marangoni number

$$
\mathrm{Ma}=\frac{\left|\frac{\partial \sigma}{\partial T}\right| \beta h^{2}}{\mu \kappa}
$$

and the Rayleigh number

$$
\mathrm{Ra}=\frac{\alpha g \beta h^{4}}{\nu \kappa},
$$

where $\beta=|\partial T / \partial x|$ is the gradient, $\alpha$ the thermal expansion coefficient, $g$ the gravity, $\sigma$ the surface tension, $\nu$ the kinematic viscosity, $\kappa$ the thermal diffusivity, and $\mu$ the dynamic viscosity. In most of the measurements Ma was greater than $\mathrm{Ra}$, so thermocapillary forces were dominant. For instance, for $h=2 \mathrm{~mm}$ Ma was twice Ra. We also considered a $\beta_{\text {eff }}$ as the constant gradient (effective gradient) in the center of the cell, far from the influence of the sidewalls. This $\beta_{\text {eff }}$ was used because the measurements were obtained in the central bulk, in which the temperature profile is linear. Through temperature measurements obtained from thermocouples placed in this region of the fluid, for a constant height $(h=2 \mathrm{~mm})$, we saw that $\mathrm{Ma} \simeq 500$ and so this value approaches the theoretical Marangoni $(\mathrm{Ma} \simeq 390)$ found by SD for an infinite length layer. As this theoretical result did not take into account the influence of the sidewalls that lead to a linearity breaking of the temperature profile near the end of the cell, a good comparison between experimental and theoretical results must be fulfilled away from the sidewalls, in the central region of the fluid. Perhaps this could be the cause of the difference existing between numerical values calculated in some articles and experimental data (here $\mathrm{Ma}$ $=1028$ for $h=2 \mathrm{~mm}$ ) found when the temperature difference in the whole cell was considered. Despite taking this into consideration, the numerical values are lower [13] than experimental ones. The numerical calculation of Ref. [3] in two dimensions provides a $\mathrm{Ma}=283$ for an aspect ratio equal to 20 considering a temperature with constant slope.

A usual discrepancy presented in experiments is the angle between the wave vector and the temperature gradient. Our results show a variable angle, which is limited between $35^{\circ}$ and $55^{\circ}$, whereas the theoretical values are smaller. SD show a variable angle according to the Prandtl number that for $\operatorname{Pr}=10$ is $\Theta=20^{\circ}$. Parmentier et al. [4] found a greater angle because of the inclusion of the buoyancy forces in their analysis. However, none of these papers studies the influence of the geometry of the container, which could be relevant in experiments.

For a constant height of fluid $h=2 \mathrm{~mm}$, temperature horizontal profiles were measured by introducing one or four thermocouples in the fluid bulk. The linearity of the mean horizontal temperatures in the core region for several levels from the surface to the bottom is observed. Each point of the profile has been calculated by making an average of the temperature fluctuations detected with the thermocouples. Temperature oscillations measured with four thermocouples at the same level near the bottom are shown in Fig. 6(a) and oscillations using only one thermocouple at several vertical levels are plotted in Fig. 6(b). The maximum amplitude of the oscillations had a value of $0.1 \mathrm{~K}$. As can be seen in Fig. 6(b), this amplitude is higher near the bottom and lower at the surface. The frequency of the oscillations is the same as that obtained by optical methods. Considering these waves as sinusoidal signals, we performed some calculations to get the maximum and minimum temperature ranges. The results are given in Fig. 7, which clearly shows that the highest amplitude is located near the bottom.

\section{DISCUSSION}

In this section we discuss and compare our results with other experimental and theoretical results calculated for $\mathrm{Pr}$ $=10$. We obtained a wave number $K=2.2$ similar to some experiments [7] and close to the value found by SD ( $K$ $=2.6$ ). The direction of the traveling waves, opposite to the direction of the surface flow, is in agreement with SD's analysis, although the experimental angle between the wave vector and the gradient is different from the theoretical value. If $\mathrm{Ra} \neq 0$ is considered [4] the angle approaches our experimental value. The analysis carried out by Mercier and Normand [12] finds an angle similar to ours when thermocapillary forces dominate buoyancy effects. However, data given in other experiments are very different. For this reason we think that the angle could be influenced by the geometry of the container. Experiments with a wide range of dimensions are currently being undertaken in order to clarify this behavior.

The dimensionless frequency $(\omega=7.4)$ for $h=2 \mathrm{~mm}$ is close to the value found by Daviaud and Vince [7], $\omega=10$. A nearby value was also obtained by Mercier and Normand from their numerical work [12]. If we carry out the same nondimensionalization used by SD, our result $\omega=0.011$ is half their value $\omega=0.02$. In addition, as has been mentioned before, the theoretical $\mathrm{Ma}$ is smaller than the Ma found in experiments. These two experimental results (a lower $\omega$ and a higher Ma) probably mean that energy is being dissipated 

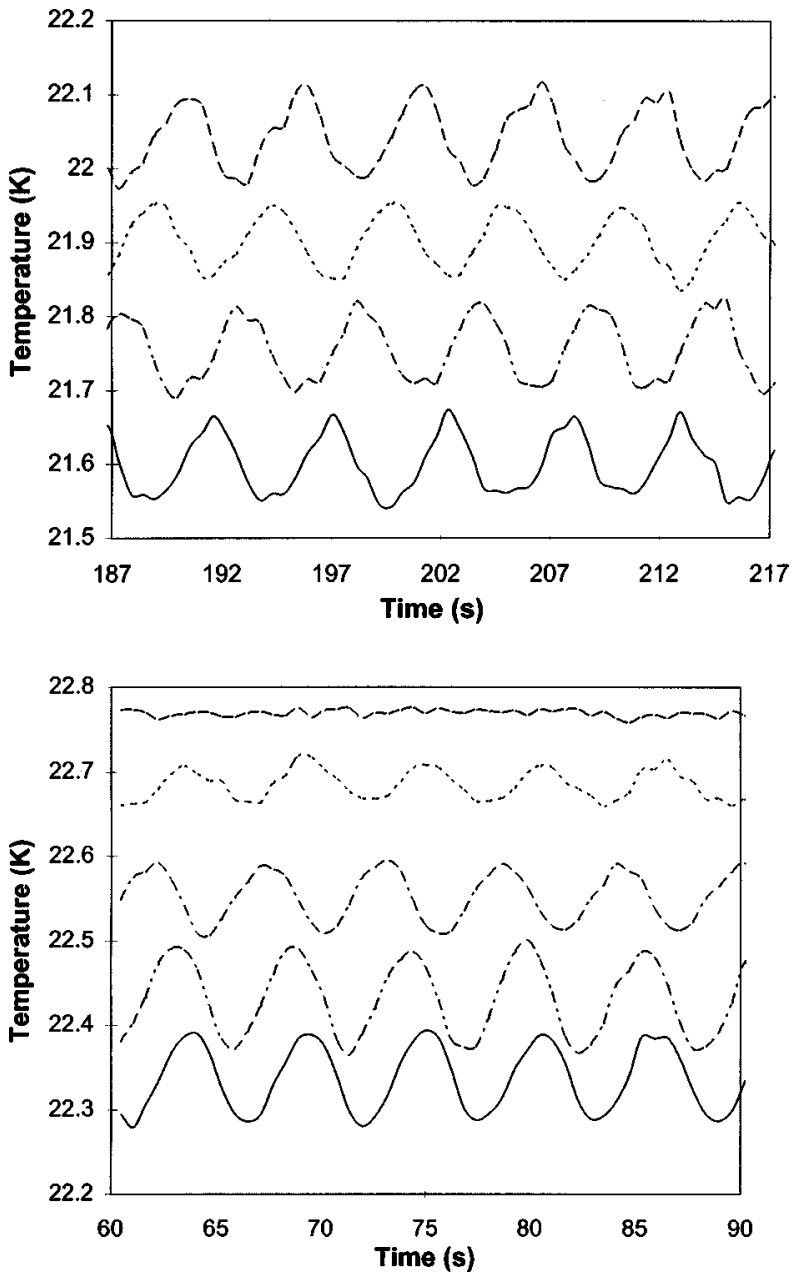

FIG. 6. Temperature oscillations for $h=2 \mathrm{~mm}$ in the central fluid bulk. (a) Using four thermocouples horizontally placed at 0.5 $\mathrm{mm}$ from the bottom. The signals correspond to thermocouples spaced $2.5 \mathrm{~mm}$ from $25 \mathrm{~mm}$, near the hot side (top signal), to 32.5 $\mathrm{mm}$ (bottom signal, which acquires the lowest temperature). Temperature fluctuation amplitude $(\simeq 0.1 \mathrm{~K})$ is similar in all of them. (b) With a thermocouple at each of five vertical levels: $2 \mathrm{~mm}$ (surface), $1.5 \mathrm{~mm}, 1 \mathrm{~mm}, 0.5$ and $0 \mathrm{~mm}$ (bottom). Data are consecutively plotted starting with surface measurements (top signal) and finishing with the temperature measurements at the bottom (bottom signal). It can be noted that the deeper the thermocouple is placed, the higher the amplitude detected.

from some part of the system, such as the surface or the bottom of the cell, which might not be as isolated as we thought. On the other hand, whereas theoretical works consider infinite systems, our experimental container has large but finite horizontal aspect ratios.

SD distinguished two types of instability for small and large Pr when the structure of basic flow is the return flow (see [15]). The instability propagates almost exactly in the spanwise direction when $\mathrm{Pr}$ is small and almost exactly upstream if $\mathrm{Pr}$ is large. For intermediate $\mathrm{Pr}$ the propagating angle would be between $80^{\circ}$ and $7.9^{\circ}$. As we showed in Sec. III, although the analysis of SD determines an angle $\Theta$ $=20^{\circ}$ for $\operatorname{Pr}=10$, the influence of the geometry of the cell on the one hand and the consideration of the buoyancy forces $(R a \neq 0)$ on the other hand would vary this value to something close to the experimental data.

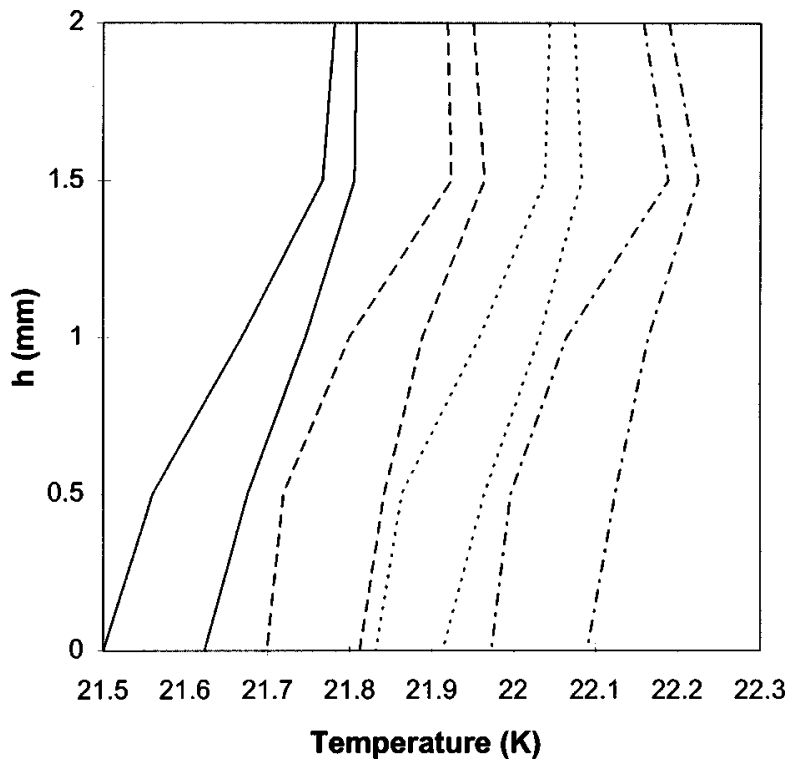

FIG. 7. Curves of the maximum and minimum temperatures of the oscillations obtained with four thermocouples horizontally spaced $2.5 \mathrm{~mm}$ and placed at each of the five height levels from the surface to the bottom. Each pair of curves with the same type of line indicates the maximum and minimum temperatures measured by a thermocouple.

The first instability mechanism (for small Pr) takes place as a result of inertia forces dominating viscous strengths, whereas in the second one (for large Pr) viscous forces dominate. In this latter case, owing to thermocapillary forces, a temperature perturbation develops, which interacts with the velocity field of the return flow and as a result an interior hot spot is produced. This hot spot heats the surface and the surrounding fluid and it moves to the hot side when the supply of energy is large enough. As a result, waves are shown traveling upstream whose temperature amplitude is higher near the bottom than at the surface.

From the isotherms of Fig. 5 in Ref. [15], for large Pr, it is not difficult to obtain the nondimensional variation of the temperature perturbation flow field in the fluid bulk according to depth. The result shows an increase in the oscillations amplitude near the bottom and a minimum value at the surface. Our experimental data present a similar behavior, which is shown in Fig. 6(b). If the perturbation field is superimposed on the vertical profile of the basic flow (Fig. 1 of Ref. [15]), traveling waves with a higher amplitude near the bottom are obtained, as was measured in this experiment (Fig. 7).

\section{CONCLUSIONS}

We obtained a wave number $K=2.2$, for $\operatorname{Pr}=10$, which is similar to that found in other experimental and theoretical works. A pair of oblique hydrothermal waves that propagate upstream, i.e., in the same direction as the temperature gradient, were observed, like those SD found. The difference between the experimental and the theoretical angle could be due to some imposition of the geometry of the cell, but this difference might be smaller because of the presence of buoyancy forces. With $l_{x}$ longer than in other experiments, a higher $\Delta T$ at the threshold has been found. Although the Ma- 
rangoni number calculated for a constant temperature gradient comes near the theoretical value, the higher experimental $\mathrm{Ma}$ and the lower frequency could mean that there is a dissipation mechanism in the system.

From the measurements obtained through thermocouples with $h=2 \mathrm{~mm}$, we observed waves with the same unique frequency $\omega=1.2 \mathrm{~Hz}$ as was obtained from the spatiotemporal diagram. Vertical and linear horizontal profiles were obtained in the fluid bulk as well as the temperature oscillations. The agreement between the amplitude, which is maximum near the bottom, and some conclusions extracted from numerical results of SD provides support for the instability mechanism suggested by these authors. Nevertheless, more measurements in the whole bulk at several depths and a container with a wide range of dimensions would enable us to obtain more general behavior.

\section{ACKNOWLEDGMENTS}

We are especially grateful to Angel Garcimartín for his help and also to H. Mancini, C. Pérez-García, and Blas Echebarria for their valuable suggestions and comments. This work was supported by the Asociación de Amigos (University of Navarra) and DGICYT (Spanish Government) through Grant No. PB95-0578.
[1] G. Müller, J. Cryst. Growth 128, 26 (1993).

[2] M. K. Smith and S. H. Davis, J. Fluid Mech. 132, 119 (1983).

[3] P. Laure and B. Roux, C. R. Acad. Sci., Ser. II: Mec. Phys., Chim., Sci. Terre Univers 305, 1137 (1987).

[4] P. M. Parmentier, V. C. Regnier, and G. Lebon, Int. J. Heat Mass Transf. 36, 9 (1993); 36, 2417 (1993).

[5] D. Villers and J. K. Platten, J. Fluid Mech. 234, 487 (1992).

[6] M. G. Braunsfurth and G. M. Homsy, Phys. Fluids 9, 1277 (1997).

[7] F. Daviaud and J. M. Vince, Phys. Rev. E 48, 4432 (1993).

[8] D. Schwabe, U. Möller, J. Schneider, and A. Scharmann, Phys. Fluids A 4, 2368 (1992).
[9] A. B. Ezersky, A. Garcimartin, H. L. Mancini, and C. PérezGarcía, Phys. Rev. E 48, 4414 (1993).

[10] R. J. Riley and G. P. Neitzel, J. Fluid Mech. 359, 143 (1998).

[11] M. K. Smith, J. Fluid Mech. 194, 391 (1988).

[12] J. F. Mercier and G. Normand, Phys. Fluids 8, 1433 (1996).

[13] J. Xu and A. Zebib, J. Fluid Mech. 364, 187 (1998).

[14] C. De Saedeleer, A. Garcimartin, G. Chavepeyer, J. K. Platten, and G. Lebon, Phys. Fluids 8, 670 (1996).

[15] M. K. Smith, Phys. Fluids 29, 3182 (1986).

[16] A. Garcimartin, N. Mukolobwiez, and F. Daviaud, Phys. Rev. E 56, 1699 (1997).

[17] J. Burguete, N. Mukolobwiez, and F. Daviaud (unpublished). 